\title{
Comparing the effectiveness of neutrophil- lymphocyte ratio as a mortality predictor on middle and advanced age coronary artery bypass graft patients
}

\author{
Derih Ay ${ }^{1}$, Burak Erdolu' ${ }^{1}$, Gunduz Yumun ${ }^{2}$, Ufuk Aydin ${ }^{1}$, Ahmet Demir ${ }^{3}$, \\ Osman Tiryakioglu ${ }^{4}$, Ahmet Hakan Vural ${ }^{1}$ \\ ${ }^{1}$ Department of Cardiovascular Surgery, Bursa Yuksek Ihtisas Education and Research Hospital, Bursa, Turkey; \\ 2Department of Cardiovascular Surgery, Namik Kemal Univercity, Medical Faculty, Tekirdag, Turkey; \\ ${ }^{3}$ Department of Cardiovascular Surgery, Yalova State Hospital, Yalova, Turkey; \\ ${ }^{4}$ Department of Cardiovascular Surgery, Bahcesehir Univercity, Medical Faculty, Medical Park Hospital, Bursa, Turkey
}

\section{ABSTRACT}

OBJECTIVE: In this study, the effect of neutrophil-lymphocyte ratio (NLR), which is a recently developed inflammatory parameter, as an early stage mortality predictive marker on coronary artery bypass (CABG) patients of various age groups was examined.

METHODS: Seventy eight patients under the age of 45 (Group 1) and 80 patients who were older than 45 (Group 2) randomly chosen from the patients who underwent isolated CABG surgery, were examined. The preoperative characteristics and NLRs were noted. The primary end point of the study was determined as all-cause in- hospital mortality.

RESULTS: Mortality was observed in 2 patients in Group 1 and 11 patients in Group 2. The threshold value of NLR was 2,47 in the Receiver Operating Characteristic (ROC) curve in Group 1 and there wasn't any significant correlation between preoperative NLR and mortality rates in the patients whose NLRs were above this curve. The threshold value was determined as 4.07 in Group 2 and there was a significant relation between preoperative NLR and mortality $(p<0,01)$. No relation was found between NLR and mortality when all the examined patients were considered $(p>0.05)$.

CONCLUSION: NLR that can be easily calculated, can be used as a mortality predictor in the patients of advanced age who will undergo isolated CABG procedure.

Key words: Biological markers; coronary artery disease; inflammation; prognosis. 
A lthough coronary artery bypass grafting (CABG) surgery is a surgical procedure described for coronary artery disease, still unpredicted mortality, and morbidity have been observed [1]. EuroSCORE is frequently accepted, and used risk assessment model in cardiac surgery [2]. Because of concerns about under-, or overestimation of the risk, more reliable markers, and scoring systems are needed.

In many studies inflammation markers, and their relationship with cardiovascular risks have been indicated [3]. White blood cell count (WBC) has been demonstrated as a predictor of mortality after CABG [4, 5]. However subtypes of WBC, and their ratios have been demonstrated as more valu- able predictors than WBC $[6,7]$. Neutrophil /lymphocyte ratio (NLR) can be estimated easily from differential cell counts. Although NLR is a marker of inflammatory conditions, it also enables combined evaluation of neutrophilia, and lymphopenia which have worse cardiovascular prognosis $[8,9]$.

We investigated NLR which is an early-phase post-CABG mortality predictor in various age groups.

\section{MATERIALS AND METHODS}

After approval of our hospital's scientific publication rating committee, 158 patients who had under-

TABLE 1. Preoperative, and operative data

\begin{tabular}{lccc} 
& Group 1 & Group 2 & P \\
\hline Clinical characteristics & & & \\
N & 78 & 80 & NS \\
Gender (F/M) & $28(35.8 \%) / 50(64.2 \%)$ & $30(37.5 \%) / 50(62.5 \%)$ & NS \\
Age (mean)years & $39.4 \pm 4.1$ & $63.9 \pm 9.2$ & 0.001 \\
EF & $48.7 \pm 10.9$ & $48.6 \pm 13.1$ & NS \\
Euroscore & $1.2 \pm 1.4$ & $3.4 \pm 2.3$ & 0.001 \\
Smoking & $40(51.2 \%)$ & $33(41.5 \%)$ & 0.490 \\
Hypertension & $38(48.7 \%)$ & $35(43.7 \%)$ & 0.748 \\
Diabetes mellitus & $27(34.7 \%)$ & $18(22.5 \%)$ & 0.122 \\
CVD & $2(2.5 \%)$ & $4(5 \%)$ & 0.317 \\
Hyperlipidemia & $27(34.7 \%)$ & $20(25 \%)$ & 0.395 \\
COPD (Moderate -Advanced) & $10(12.8 \%)$ & $13(16.3 \%)$ & 0.523 \\
Laboratory & & & \\
Urea & $23.2 \pm 10.7$ & $37.5 \pm 12.9$ & 0.001 \\
Creatinine & $0.9 \pm 0.6$ & $2.3 \pm 1.3$ & NS \\
WBC & $9.2 \pm 2.5$ & $9.7 \pm 10.6$ & NS \\
Neutrophil & $5.8 \pm 2.1$ & $6.2 \pm 5.2$ & NS \\
Lymphocyte & $2.4 \pm 0.7$ & $1.9 \pm 0.8$ & 0.001 \\
N/L ratio (mean ) & 2.47 & 4.07 & 0.001 \\
Operatif & & & \\
Duration of CPB (min) & $70.3 \pm 44.9$ & $93.6 \pm 34.6$ & 0.001 \\
Duration of CC (min) & $52.9 \pm 37.6$ & $67.1 \pm 26.7$ & 0.007 \\
CABG (number of grafts) & $2.3 \pm 0.9$ & $3.0 \pm 0.8$ & 0.001 \\
Mortality (1 month) & 2 & 11 & 0.001 \\
\hline
\end{tabular}

EF: Ejection Fraction; CVD: Cardiovascular Disease; COPD: Chronic Obstructive Pulmonary Disease; WBC: Total White Blood Cell Count; CPB: Cardiopulmonary Bypass; CC: Cross-Clamping; CABG: Coronary Artery Bypass Grafting; NS: Not Significant P<0.05 significant. 


\begin{tabular}{|c|c|c|c|}
\hline & $\begin{array}{c}\text { Increased N/L } \\
51\end{array}$ & $\begin{array}{c}\text { Decreased N/L } \\
107\end{array}$ & $P$ \\
\hline Age (mean) & $50.4 \pm 15.2$ & $52.5 \pm 13.7$ & NS \\
\hline $\mathrm{EF}$ & $49.1 \pm 10.7$ & $48.4 \pm 12.6$ & NS \\
\hline EuroSCORE & $2.5 \pm 2.5$ & $2.2 \pm 2.0$ & NS \\
\hline Urea & $31.0 \pm 14.9$ & $30.1 \pm 13.3$ & NS \\
\hline Creatinine & $1.1 \pm 0.8$ & $1.9 \pm 1.3$ & NS \\
\hline WBC & $10.5 \pm 3.8$ & $9.0 \pm 4.6$ & NS \\
\hline Neutrophil & $7.9 \pm 3.2$ & $5.1 \pm 4.0$ & 0.0001 \\
\hline Lymphocyte & $1.7 \pm 0.7$ & $2.4 \pm 0.8$ & 0.0001 \\
\hline CPB & $74.7 \pm 40.9$ & $85.7 \pm 41.6$ & NS \\
\hline CC & $52.8 \pm 28.9$ & $63.6 \pm 34.6$ & NS \\
\hline CABG & $2.5 \pm 0.9$ & $2.8 \pm 0.9$ & NS \\
\hline Mortality & 7 & 6 & NS \\
\hline
\end{tabular}

gone isolated on-pump CABG operation between 2011, and 2014 by the same surgical team were retrospectively evaluated. The patients were divided into 2 groups as 78 patients of $\leq 45$ years of age (Group 1), and 80 randomly selected patients aged over 45 years (Group 2). Routine biochemical, and hematological values of the patients were recorded. Their pulmonary functions, medications used, presence of diabetes mellitus, and concomitant diseases were investigated, and together with their cardiac data EuroSCORE values were calculated (Table 1).

Preoperative demographic characteristics, total white blood cell count, neutrophil ratios, lymphocyte ratios, NLR, cross-clamping, and cardiopulmonary bypass times were recorded (Table 2). Primary endpoint was observation of all-cause in-patient mortality. Mean NLR values were calculated separately for each group (Table 3 ).

\section{Statistical analysis}

The patients included in the study were analyzed in two separate groups, and 4 subgroups, and values were expressed as mean \pm standard deviation. Parametric data were evaluated with t-test, and non-parametric data using chi-square test. In the evaluation of characteristics effective on mortality, Pearson's two-way correlation test was used. $\mathrm{P}<0.05$ was accepted as statistically significant.

\section{RESULTS}

Estimated threshold NLR values were 2.47 for Group 1, and 4.07 for Group 2. In Group 1, a significant correlation was not detected between mortality rates, and NLR values in patients whose NLR values were above threshold values $(p<0.05)$. In Group 2, a significant correlation was detected between mortality rates, and NLR values in patients whose NLR values were above threshold values $(p<0.01)$. When all patients included in the study were evaluated, a significant correlation was not found between NLR values, and mortality rates $(\mathrm{p}<0.05)$. In addition, a statistically significant correlation existed between EuroSCORE scale scores, and mortality rates. Higher mortality rates were detected in patients with higher EuroSCORE scale scores $(p=0.0001)$. However any relation of this association with NLR values was not observed. $(p>0.05)$. Only in the group with higher EuroS- 


TABLE 3. Variables effective on mortality
\begin{tabular}{lccc} 
Variables & Deceased (13) & Living (145) & P \\
\hline Inotropic use & 13 & 77 & 0.001 \\
IABP & 6 & 8 & 0.001 \\
Prolonged ventilation & 6 & 11 & 0.004 \\
Duration of CPB & $104(20-207)$ & $81(0-262)$ & 0.041 \\
EuroScore & $5(3-9)$ & $2(0-9)$ & 0.001 \\
NLR & $4.28(2-14)$ & $2.33(1-21)$ & 0.001 \\
Lymphocyte & $1.6(1-3)$ & $2.1(0-5)$ & 0.001 \\
Age (years) & $62.6 \pm 14$ & $50.8 \pm 13.8$ & 0.004 \\
\hline
\end{tabular}

IABP: Intraaortic Balloon Pump; CPB: Cardiopulmonary Bypass;

NLR: Neutrophil/Lymphocyte Ratio.

CORE scale scores left ventricular ejection fraction (EF) was effective on mortality $(p=0.0001)$. However age, NLR, and even lower EF values had no effect on mortality rates (Table 2). Difference detected between creatinine levels of both two group were not statistically significant. This difference in creatinine levels might trigger inflammatory processes with resultant higher NLR values. However as stated above this difference was not statistically significant.

\section{Exclusion criteria}

Patients who underwent off-pump surgery, concurrent valvular surgery, urgently operated cases, those with acute or chronic infections, known malignancies, and hematological problems were not included in the study.

\section{DISCUSSION}

The main purpose of developing EuroSCORE system was to measure the quality of cardiac surgical procedures [10]. However following its introduction into medical practice, inadequacities of the $\mathrm{Eu}$ roSCORE system have been reported [11]. Later on, another scoring system by The Society of Thoracic Surgeons (STS) was described, and published [12]. STS scoring system which is more detailed relative to the EuroSCORE system has demon- strated its superiority over EuroSCORE in many cardiac surgery groups [13-15]. However clinical application of STS scoring system is very difficult. Therefore, in our clinic we are using EuroSCORE system for risk assessments. In our survey, we have detected higher EuroSCORE values in patients with increased risk of mortality. However higher mortality rates have been evaluated independent from increases in NLRs.

The primary purpose of using risk scoring systems, and biopredictors is to predict unwanted intraoperative conditions. In addition to EuroSCORE systems, new markers have been defined. Total white blood cell count was the first biopredictor found to be correlated with mortality. Bagger et al. performed a study on 2058 patients, and determined WBC as a predictor of post-CABG 30-day mortality [4]. Besides, Newall et al. in their series consisting of 3024 patients, detected a correlation between preoperative $\mathrm{WBC}$, and perioperative myocardial injury, and 1-year mortality [5]. Despite the outcomes of such a large series, it is known that white blood cell count is a nonspecific marker which can increase due to various conditions. For this reason, it is not reliable to use this marker by itself. Contrary to these studies, Gibson et al. analyzed specific cell counts, and ratios in a study population of 1938 patients, and couldn't find a correlation between $\mathrm{WBC}$, and mortality rates [8]. 
Recently, NLR has become prominent as a biomarker. As an indicator of inflammatory state, combination of netrophilia, and lymphopenia has been associated with poor cardiovascular prognosis [16]. Increase in the number of neutrophils is an indicator of active inflammatory process, while decrease in the number of lymphocytes is an indicator of inadequacy of the active inflammatory process. Correlation between lymphopenia, and progression of atherosclerosis, and major cardiac complications has been demonstrated $[17,18]$. Neutrophils induce formation of reactive oxygen radicals, and inflammatory mediators in myocardium during $\mathrm{CPB}$ [19]. In large series, it is possible to correlate preoperative NLR, and post-CABG mortality rates. Gibson et al. pioneered this assumption. A correlation between preoperative NLR derived from WBC and mortality rates exists which is independent from well-known personal factors, and EuroSCORE values [8]. In a study performed by Ünal et al,, a correlation was detected between preoperative NLR, and post-CABG mortality rates [20]. However in our study among young patient group an association between NLR, and mortality was not detected, however a correlation was found between NLR, and mortality in the elder patient group. When all patients were considered, a correlation was not detected between NLR, and mortality rates.

Limitations of the study: A single-centered investigation was performed on a small patient group which can be considered as an important limitation of our study. Apart from many clinical parametres we evaluated, unknown factors as inflammatory responses induced by cardiac injury during clinical course, and cardiopulmonary bypass might effect accuracy of our outcomes.

\section{CONCLUSION}

We think that, as an easily calculable post-CABG biopredictor of mortality with low cost, NLR together with EuroSCORE scoring system can be used in the elder population. In our study, higher NLO value has been demonstrated as a marker of mortality. However for quantitative determination of the risk of mortality based on NLR values, larg- er-scale studies should be conducted.

Conflict of Interest: No conflict of interest was declared by the authors.

Financial Disclosure: The authors declared that this study has received no financial support.

\section{REFERENCES}

1. Møller CH, Penninga L, Wetterslev J, Steinbrüchel DA, Gluud C. Off-pump versus on-pump coronary artery bypass grafting for ischaemic heart disease. Cochrane Database Syst Rev 2012;3:CD007224.

2. Siregar S, Groenwold RH, de Heer F, Bots ML, van der Graaf Y, van Herwerden LA. Performance of the original EuroSCORE. Eur J Cardiothorac Surg 2012;41:746-54.

3. Pearson TA, Mensah GA, Alexander RW, Anderson JL, Cannon RO 3rd, Criqui M, et al. Markers of inflammation and cardiovascular disease: application to clinical and public health practice: $\mathrm{A}$ statement for healthcare professionals from the Centers for Disease Control and Prevention and the American Heart Association. Circulation 2003;107:499-511.

4. Bagger JP, Zindrou D, Taylor KM. Leukocyte count: a risk factor for coronary artery bypass graft mortality. Am J Med 2003;115:660-3.

5. Newall N, Grayson AD, Oo AY, Palmer ND, Dihmis WC, Rashid A, et al. Preoperative white blood cell count is independently associated with higher perioperative cardiac enzyme release and increased 1-year mortality after coronary artery bypass grafting. Ann Thorac Surg 2006;81:583-9.

6. Grau AJ, Boddy AW, Dukovic DA, Buggle F, Lichy C, Brandt T, et al. Leukocyte count as an independent predictor of recurrent ischemic events. Stroke 2004;35:1147-52.

7. Horne BD, Anderson JL, John JM, Weaver A, Bair TL, Jensen $\mathrm{KR}$, et al. Which white blood cell subtypes predict increased cardiovascular risk? J Am Coll Cardiol 2005;45:1638-43.

8. Gibson PH, Cuthbertson BH, Croal BL, Rae D, El-Shafei H, Gibson $\mathrm{G}$, et al. Usefulness of neutrophil/lymphocyte ratio as predictor of new-onset atrial fibrillation after coronary artery bypass grafting. Am J Cardiol 2010;105:186-91.

9. Madjid M, Awan I, Willerson JT, Casscells SW. Leukocyte count and coronary heart disease: implications for risk assessment. J Am Coll Cardiol 2004;44:1945-56.

10. Nashef SA, Roques F, Michel P, Gauducheau E, Lemeshow S, Salamon R. European system for cardiac operative risk evaluation (EuroSCORE). Eur J Cardiothorac Surg 1999;16:9-13.

11. Gogbashian A, Sedrakyan A, Treasure T. EuroSCORE: a systematic review of international performance. Eur J Cardiothorac Surg 2004;25:695-700.

12. The Society of Thoracic Surgeons. Executive Summary: Society of Thoracic Surgeons Spring 2007 report. Chicago, IL: The Society of Thoracic Surgeons; 2007. 
13. Metzler B, Winkler B. SYNTAX, STS and EuroSCORE - how good are they for risk estimation in atherosclerotic heart disease? Thromb Haemost 2012;108:1065-71.

14. Wendt D, Osswald BR, Kayser K, Thielmann M, Tossios P, Massoudy P, et al. Society of Thoracic Surgeons score is superior to the EuroSCORE determining mortality in high risk patients undergoing isolated aortic valve replacement. Ann Thorac Surg 2009;88:468-75.

15. Kunt AG, Kurtcephe M, Hidiroglu M, Cetin L, Kucuker A, Bakuy V, et al. Comparison of original EuroSCORE, EuroSCORE II and STS risk models in a Turkish cardiac surgical cohort. Interact Cardiovasc Thorac Surg 2013;16:625-9.

16. Gibson PH, Cuthbertson BH, Croal BL, Rae D, El-Shafei H, Gibson G, et al. Usefulness of neutrophil/lymphocyte ratio as predictor of new-onset atrial fibrillation after coronary artery bypass grafting. Am J Cardiol 2010;105:186-91.
17. Major AS, Fazio S, Linton MF. B-lymphocyte deficiency increases atherosclerosis in LDL receptor-null mice. Arterioscler Thromb Vasc Biol 2002;22:1892-8.

18. Núñez J, Sanchis J, Bodí V, Núñez E, Mainar L, Heatta AM, et al. Relationship between low lymphocyte count and major cardiac events in patients with acute chest pain, a non-diagnostic electrocardiogram and normal troponin levels. Atherosclerosis 2009;206:251-7.

19. Zahler S, Massoudy P, Hartl H, Hähnel C, Meisner H, Becker BF. Acute cardiac inflammatory responses to postischemic reperfusion during cardiopulmonary bypass. Cardiovasc Res 1999;41:722-30.

20. Ünal EU, Durukan AB, Özen A, Kubat E, Kocabeyoglu SS, Yurdakok O, et al. Nutrophil / lymphocyte ratio as a mortality predictor following coronary artery bypass graft surgery. Turk Gogus Kalp Damar 2013;21:588-93. 